Military Technical College Kobry El-Kobbah, Cairo, Egypt

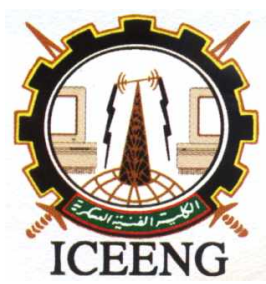

\section{$6^{\text {th }}$ International Conference on Electrical Engineering ICEENG 2008}

\title{
Circular symmetric coupled Microstrip transmission lines
}

\author{
By \\ MOHAMMAD KHALAJ-AMIRHOSSEINI* \\ AHMAD CHELDAVI*
}

\section{$\underline{\text { Abstract: }}$}

The Circular Symmetric Coupled Microstrip Transmission Lines (CSCMTL) are introduced as a new kind of coupled microstrip transmission lines. A simple method is proposed to determine the capacitance matrix of CSCMTLs. In this method the laplace's equation is solved analytically using the Fourier series expansion. Finally, the properties of CSCMTLs and the validation of the proposed method are studied using an example.

\section{Keywords:}

Coupled Microstrip Transmission Lines, Capacitance Matrix, Circular Symmetric, Laplace's equation, Fourier series Expansion

* College of Electrical Engineering, Iran University of Science and Technology, TEHRAN - I. R. IRAN 


\section{Introduction:}

The Circular Symmetric Coupled Microstrip Transmission Lines (CSCMTL), as shown in Fig. 1, could be a new kind of microstrip transmission lines in the future. The geometry of the structure includes $N$ similar circular strip lines on a cylinder with radius $b$. An internal cylinder with radius $a$ is used as the ground and the space between it and the strips is filled by a dielectric with permittivity er. The CSCMTLs are less sensitive to external electromagnetic interference and also can be designed as large scale and low crosstalk interconnects. To analyze the multiconductor transmission lines such as CSCMTLs, it is necessary to know the per-unit-length capacitance and inductance matrices [1]. The capacitance matrix of lossless structures can be determined using conformal mapping transformations [2]-[3], variational methods [4]-[5], spectral domain techniques [6]-[7], method of moments and green's function [8]-[9] and solving Laplace's equation [10]-[11]. In this paper, the capacitance matrix of CSCMTLs is determined simply by solving Laplace's equation using fourier series method. The solutions are exact but they are expressed by means of infinite linear equations. Using these methods one can determine the voltage and current distribution on the strips, also. Finally, the properties of CSCMTLs and the validation of the proposed method are studied using an example. 


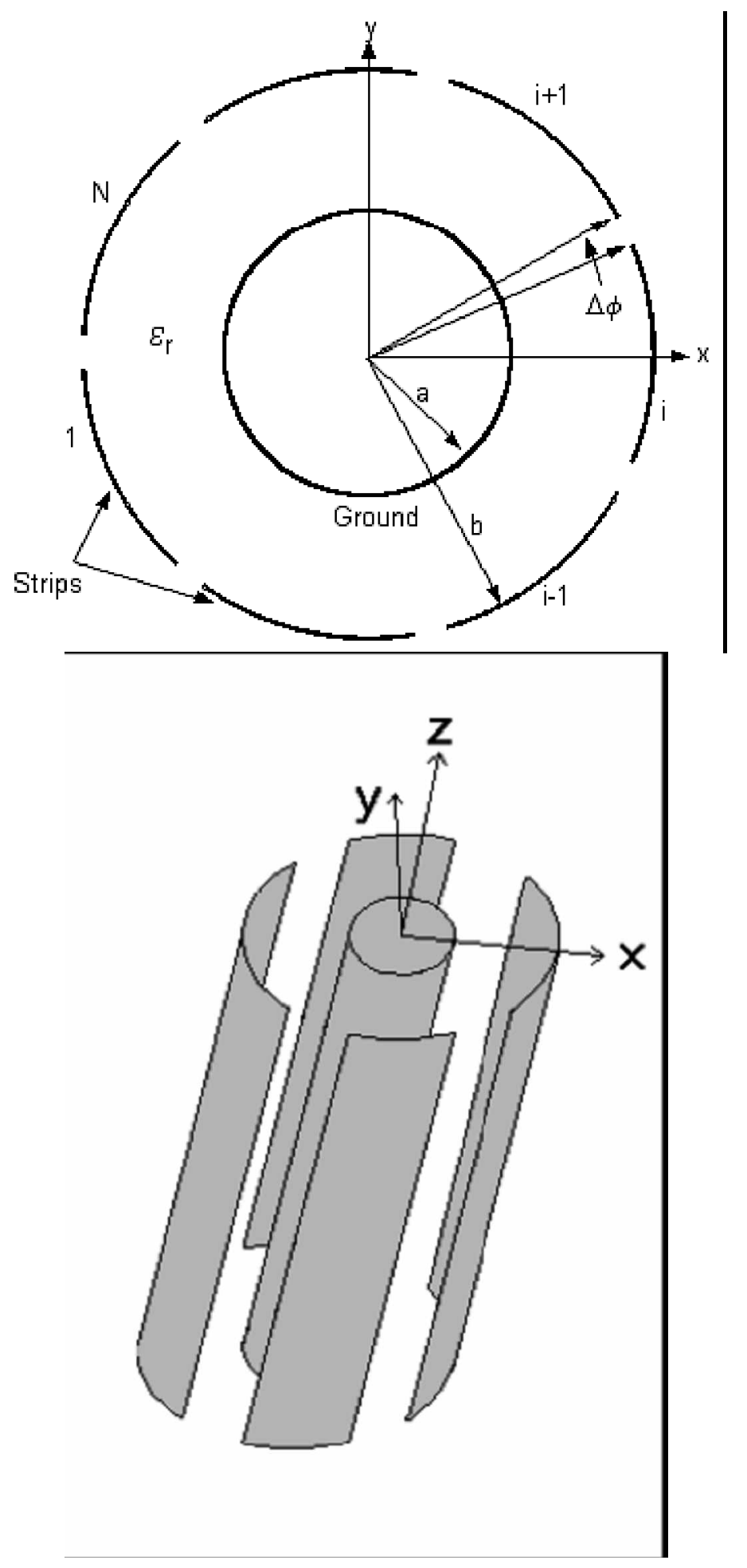

Figure (1): The cross section and longitudinal view of a typical CSCMTL 


\section{Analytic Solution of Laplace's Equation:}

In this section the capacitance and inductance matrices of CSCMTLs are determined analytically. We consider three assumption: the principal propagation mode of the structure is quasi-TEM, the strips and ground lines have perfect conductivity and the substrate is lossless. Using the boundary conditions $V(a, \mathrm{j})=V(¥, \mathrm{j})=0$, the voltage formula can be determined given by

$$
\begin{aligned}
& V_{1}(r, \varphi)=\sum_{n=1}^{\infty} A_{n}\left(r^{n}-\frac{a^{2 n}}{r^{n}}\right) \cos (n \varphi)+A_{0} \ln \left(\frac{r}{a}\right) \\
& a \leq r \leq b \text { and } \\
& V_{2}(r, \varphi)=\sum_{n=1}^{\infty} \frac{B_{n}}{r^{n}} \cos (n \varphi)+B_{0}
\end{aligned}
$$

for $r^{3} b$. From the continuity of the voltage on the surface $\mathrm{r}=b$, we will have:

$$
B_{n}=\left(b^{2 n}-a^{2 n}\right) A_{n}
$$

and

$$
B_{0}=\ln (b / a) A_{0}
$$

The surface charge on the boundary $r=b$ becomes as follows:

$$
\begin{aligned}
& \rho_{s}(\varphi)=\hat{a}_{r} .\left(\vec{D}_{2}-\vec{D}_{1}\right)=\varepsilon_{0}\left(\left.\varepsilon_{r} \frac{\partial V_{1}}{\partial r}\right|_{r=b}-\left.\frac{\partial V_{2}}{\partial r}\right|_{r=b}\right) \\
& =\sum_{n=1}^{\infty} G_{n} A_{n} \cos (n \varphi)+G_{0} A_{0} \\
& \text { where } \\
& G_{n}=\frac{\varepsilon_{0}}{b} n \frac{b^{2 n}-a^{2 n}}{b^{n}}\left(\frac{b^{2 n}+a^{2 n}}{b^{2 n}-a^{2 n}} \varepsilon_{r}+1\right) \\
& G_{0}=\frac{\varepsilon_{0}}{b} \varepsilon_{r}
\end{aligned}
$$


We assume that the voltages of the $i$-th strip and the other strips be $V 0$ and zero, respectively. Also, we know that the surface charge is zero out of the strips. Therefore, to find the unknown coefficients $A$, one can use the following relation

$$
\begin{aligned}
& \int_{\text {On the Strips }} V(b, \varphi) \cos (m \varphi) d \varphi+\int_{\text {Out of Strips }}^{-} \rho_{s}(\varphi) \cos (m \varphi) d \varphi \\
& =\int_{-\frac{\pi}{N}+\frac{\Delta \varphi}{2}}^{\frac{\pi}{N}} \frac{\Delta \varphi}{2} V_{0} \cos (m \varphi) d \varphi \quad m=0,1,2, \cdots
\end{aligned}
$$

Substituting (1) and (5) in (8), gives us:

$$
\begin{aligned}
& \sum_{n=1}^{\infty}\left[A_{n} \frac{\left(b^{2 n}-a^{2 n}\right)}{b^{n}} \sum_{k=0}^{N-1}\left(\int_{(2 k-1) \frac{\pi}{N}+\frac{\Delta \varphi}{2}}^{(2 k+1) \frac{\pi}{N} \frac{\Delta \varphi}{2}} \cos (m \varphi) \cos (n \varphi) d \varphi\right)\right] \\
& +A_{0} \ln (b / a) \sum_{k=0}^{N-1}\left(\int_{(2 k-1) \frac{\pi}{N}+\frac{\Delta \varphi}{2}}^{(2 k+1) \frac{\pi}{N} \frac{\Delta \varphi}{2}} \cos (m \varphi) d \varphi\right) \\
& +\sum_{n=1}^{\infty}\left[A _ { n } G _ { n } \sum _ { k = 0 } ^ { N - 1 } \left(\int_{(2 k+1) \frac{\pi}{N} \frac{\Delta \varphi}{2}}^{(2 k+1) \frac{\pi}{N}+\frac{\Delta \varphi}{2}} \frac{\cos (m \varphi) \cos (n \varphi) d \varphi)]}{\frac{\pi}{2}}\right.\right. \\
& +A_{0} G_{0} \sum_{k=0}^{N-1}\left(\int_{(2 k+1) \frac{\pi}{N}}^{(2 k+1) \frac{\pi}{N}+\frac{\Delta \varphi}{2}} \frac{\Delta \varphi}{2} \frac{\Delta \varphi}{2}\right.
\end{aligned}
$$

for $m=0,1, .2, \ldots$.

It is provable that 


$$
\begin{aligned}
& \sum_{k=0}^{N-1}\left(\int_{(2 k+1) \frac{\pi}{N}}^{(2 k+1) \frac{\pi}{N}+\frac{\Delta \varphi}{2}} \cos (l \varphi) d \varphi\right) \\
& =N \Delta \varphi \operatorname{sinc}\left(l \frac{\Delta \varphi}{2 \pi}\right)(-1)^{l / N} \delta(\operatorname{rem}(l / N))
\end{aligned}
$$

and

$$
\begin{aligned}
& \sum_{k=0}^{N-1}\left(\int_{(2 k-1) \frac{\pi}{N}+\frac{\Delta \varphi}{2}}^{\cos (l k)} \frac{\pi \varphi}{2}\right. \\
& = \begin{cases}-N \Delta \varphi \operatorname{sinc}\left(l \frac{\Delta \varphi}{2 \pi}\right)(-1)^{l / N} \delta(\operatorname{rem}(l / N)) ; & l \neq 0 ; \\
2 \pi-N \Delta \varphi & l=0\end{cases}
\end{aligned}
$$

in which "rem(.)" represents the remainder of its argument.

Defining $r=N \mathrm{Dj} /(2 \mathrm{p}) h$ as the ratio of total separations between the strips to the peripheral of the circle $r=b$ and after some manipulations using (9)-(11), two following relations are obtained for $m=1, .2,3, \ldots$

$$
\begin{aligned}
& A_{0}\left(r_{h} G_{0}+\left(1-r_{h}\right) \ln (b / a)\right) \\
& +r_{h} \sum_{n=1}^{\infty}\left(A_{n}\left(G_{n}-\frac{b^{2 n}-a^{2 n}}{b^{n}}\right) \sin \left(\frac{n}{N} r_{h}\right)(-1)^{n / N} \delta(\operatorname{rem}(n / N))\right) \\
& =\frac{1}{N}\left(1-r_{h}\right) V_{0}
\end{aligned}
$$

and 


$$
\begin{aligned}
& 2 r_{h} A_{0}\left(G_{0}-\ln (b / a)\right) \sin \alpha\left(\frac{m}{N} r_{h}\right)(-1)^{m / N} \delta(\operatorname{rem}(m / N)) \\
& +A_{m} \frac{b^{2 m}-a^{2 m}}{b^{m}} \\
& \left.+r_{h} \sum_{n=1}^{\infty}\left[\begin{array}{l}
A_{n}\left(G_{n}-\frac{b^{2 n}-a^{2 n}}{b^{n}}\right) \\
\left(\sin \alpha\left(\frac{m+n}{N} r_{h}\right)(-1)^{(m+n) / N} \delta\left(\operatorname{rem}\left(\frac{m+n}{N}\right)\right)\right) \\
+\sin \left(\frac{m-n}{N} r_{h}\right)(-1)^{(m-n) / N} \delta\left(\operatorname{rem}\left(\frac{m-n}{N}\right)\right)
\end{array}\right)\right] \\
& =\frac{2}{N}\left(1-r_{h}\right) \sin \left(\frac{m}{N}\left(1-r_{h}\right)\right) V_{0}
\end{aligned}
$$

Now, we can determine the unknown coefficients $A$, assuming that $n, m £ M$. With this assumption, the relations (12) and (13) can be written in a matrix form as follows:

$$
\mathbf{B}_{(M+1) \times(M+1)} A_{(M+1) \times 1}=V_{(M+1) \times 1}
$$

where $\mathbf{A}=\left[A_{0}, A_{1}, A_{2}, \ldots A_{M}\right]^{T}$ and the elements of $\mathbf{B}$

and the elements of $\mathrm{B}$ and $\mathrm{V}$ are Determined from (12)-(13). It is evident that as the number of harmonies, $M$, increases the unknown coefficients are obtained with lesser error. According to arguments of the sinc functions in equations (12) and (13), $M$ must be several times of $N / r h$. In fact this is because as $N$ increase or $r h$ decreases, the slop of boundary functions and therefore the number of necessary harmonics increases.

\section{Capacitance and Inductance Matrices:}

After finding the unknown coefficients of voltage, we can now calculate the capacitance and inductance matrices of the circular microstrip lines. Each element of the matrix $\mathrm{C}$, e.g. $\mathrm{C}(j, i)$, can be determined from total surface charge of the $j$-th strip, when the $i$-th strip is hold on the voltage $V 0$ and the other strips are hold to zero voltage. Thus using (5), we have 


$$
\begin{aligned}
& \mathrm{C}(j, i)=\frac{1}{V_{0}} \int_{(j-i-0.5)}^{(j-i+0.5)} \frac{2 \pi}{N} \rho_{s}(\varphi) b d \varphi=\frac{\Delta \varphi}{2} \\
& \times\left\{G_{0} A_{0}+\sum_{n=1}^{\infty}\left[G_{n} A_{n} \cos \left(2 \pi(j-i) \frac{n}{N}\right) \operatorname{sinc}\left(\frac{n}{N}\left(1-r_{h}\right)\right)\right]\right\}
\end{aligned}
$$

for $i$ and $j=1,2, \ldots, N$. Also the inductance matrix $\mathrm{L}$ is obtained from [1]

$$
\mathbf{L}=\frac{1}{c^{2}} \mathbf{C}_{0}^{-1}
$$

where $c$ is the velocity of the light and $\mathrm{C} 0$ is capacitance matrix assuming that $\mathrm{e} r=1$.

\section{Example and Results:}

In this section we calculate the capacitance and inductance matrices of a CSCMTL using the obtained relations. Consider a CSCMTL with the parameters $\mathrm{N}=4, \mathrm{a}=0.1$ $\mathrm{mm}, \mathrm{b}=1.0 \mathrm{~mm}$, e $\mathrm{r}=2.54, \mathrm{rh}=0.5$ and $\mathrm{V} 0=1$. Figs. 2-4 show the coefficients An, and the functions $\mathrm{V}(\mathrm{b}, \mathrm{j})$ and $\mathrm{r}(\mathrm{j}) \mathrm{s}$, respectively. We see the satisfying of the boundary conditions on four 45 o strips and between them. So one may satisfy about the validation of the obtained relations. Also, the capacitance and inductance matrices of the structure have been calculated as follows:

$$
\begin{aligned}
\mathbf{C} & =\left[\begin{array}{cccc}
41.57 & -11.99 & -3.07 & -11.99 \\
-11.99 & 41.57 & -11.99 & -3.07 \\
-3.07 & -11.99 & 41.57 & -11.99 \\
-11.99 & -3.07 & -11.99 & 41.57
\end{array}\right][p F / m] \\
\mathbf{L} & =\left[\begin{array}{llll}
777.7 & 399.3 & 335.8 & 399.3 \\
399.3 & 777.7 & 399.3 & 335.8 \\
335.8 & 399.3 & 777.7 & 399.3 \\
399.3 & 335.8 & 399.3 & 777.7
\end{array}\right][\mathrm{nH} / \mathrm{m}]
\end{aligned}
$$

It is seen that these matrices are Reciprocal, Toeplitz and Cyclic. Figs. 5-6 show the effect of the parameters $a$ and $r h$ in the capacitance coefficients. We see that as the 
radius of the ground, $a$, increases, the self capacitances are increased and the induction capacitances are decreased and consequently the coupling or the crosstalks between the lines are decreased. Also as expected, as the ratio $r h$ increases, all capacitance coefficients are decreased.

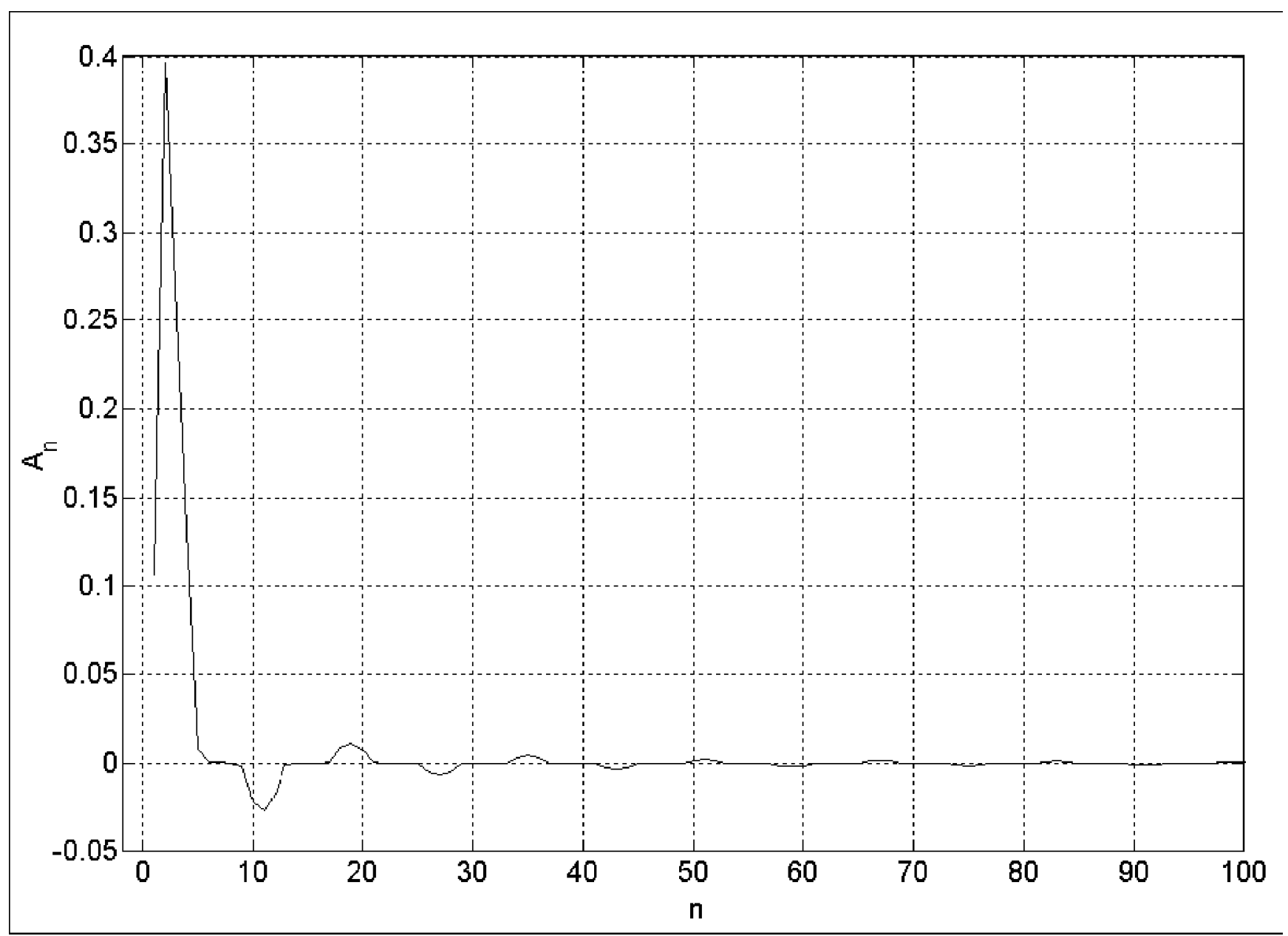

Figure (2): The unknown coefficients An 


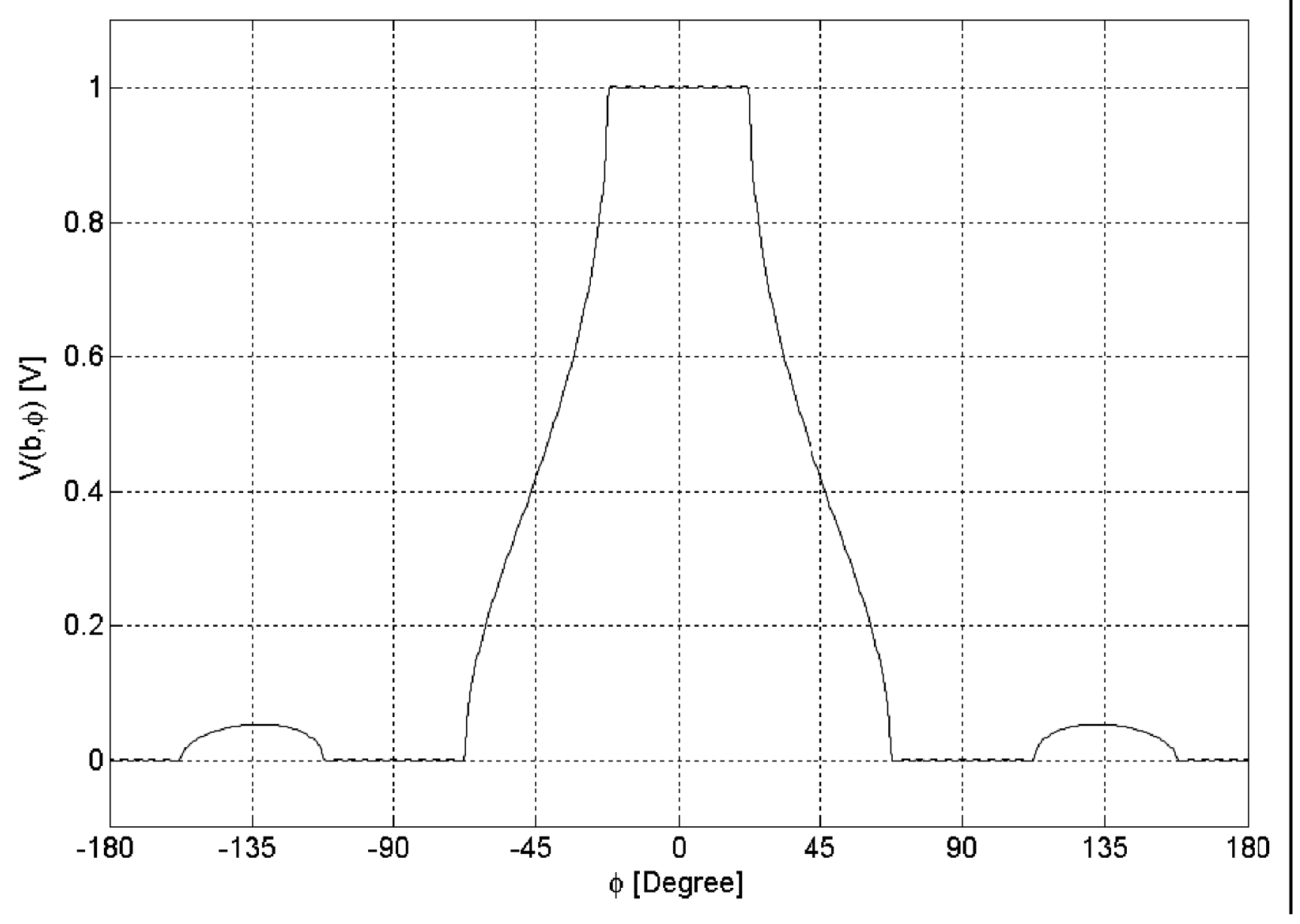

Figure (3): The voltage of the boundary $r=b$

\section{Conclusions:}

The Circular Symmetric Coupled Microstrip Transmission Lines (CSCMTL) were introduced as a new kind of coupled microstrip transmission lines. A simple method was proposed to determine the capacitance matrix of CSCMTLs. In this method the laplace's equation is solved analytically using the Fourier series expansion. The properties of CSCMTLs and the validation of the proposed method were studied using an example. The CSCMTLs are less sensitive to external electromagnetic interference and also can be designed as large scale and low crosstalk interconnects. It was seen that as the radius of the ground increases, the self capacitances are increased and the induction capacitances are decreased and consequently the coupling or the crosstalks between the lines are decreased. 


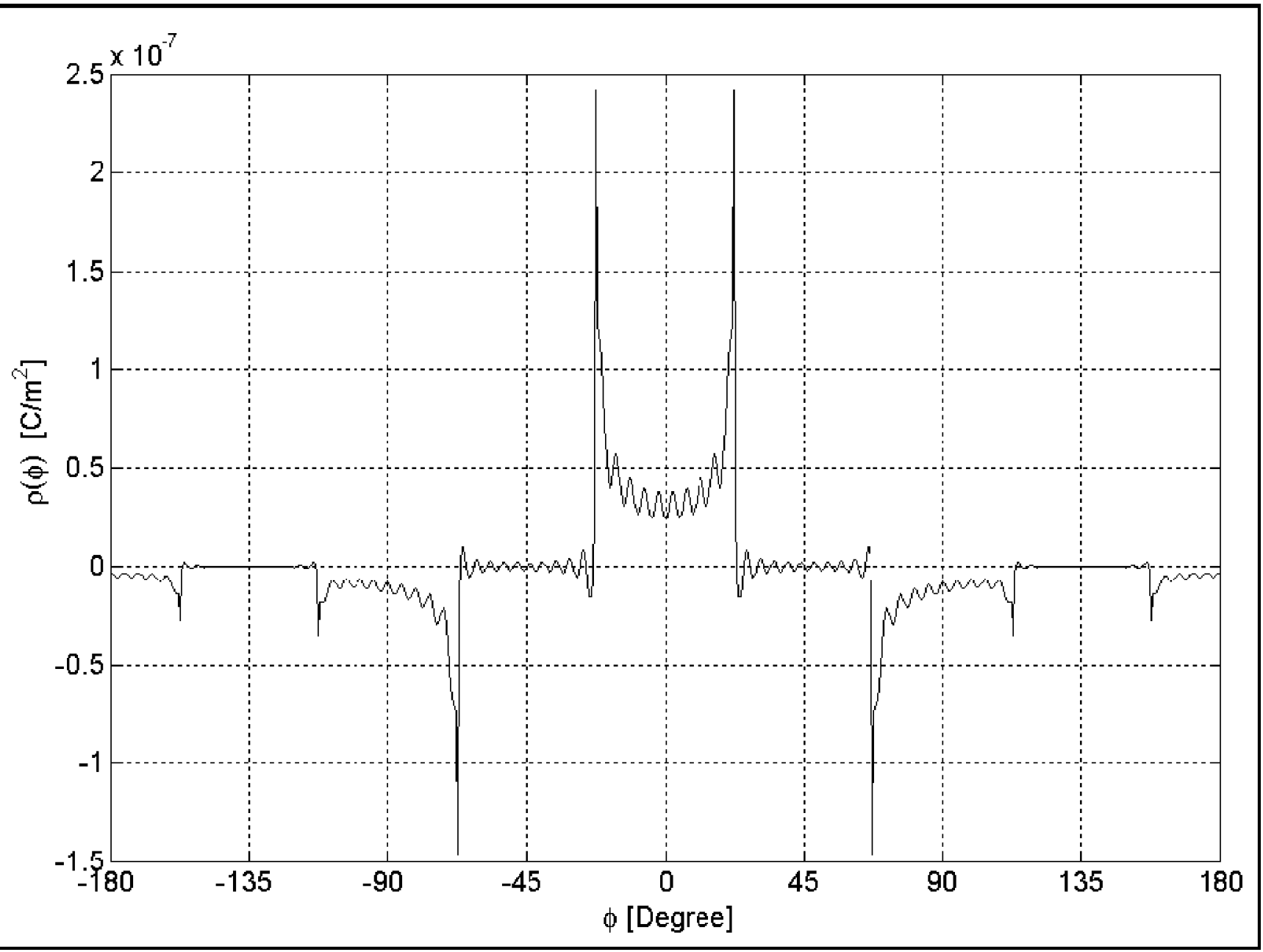

Figure (4): The surface charge on the boundary $r=b$ 


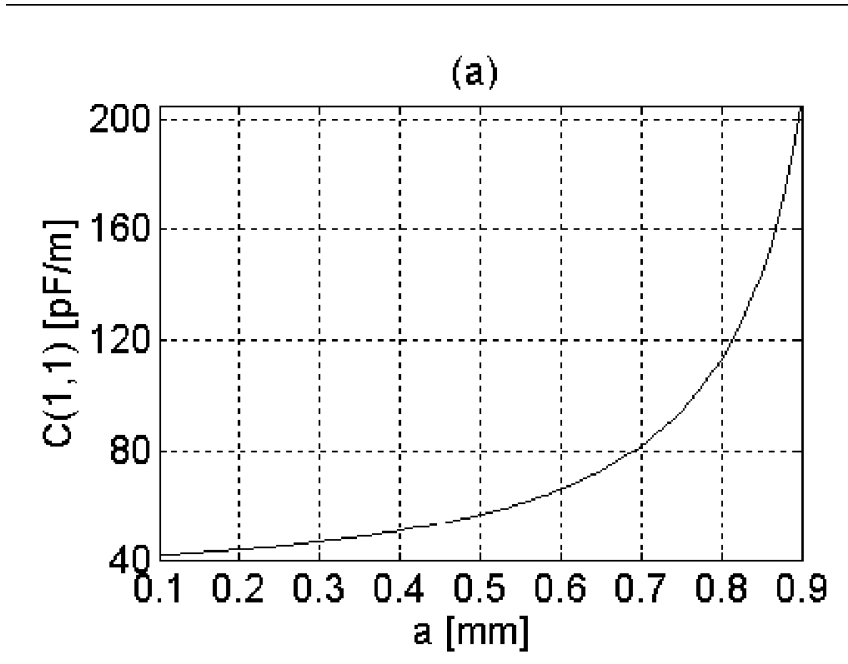

(c)

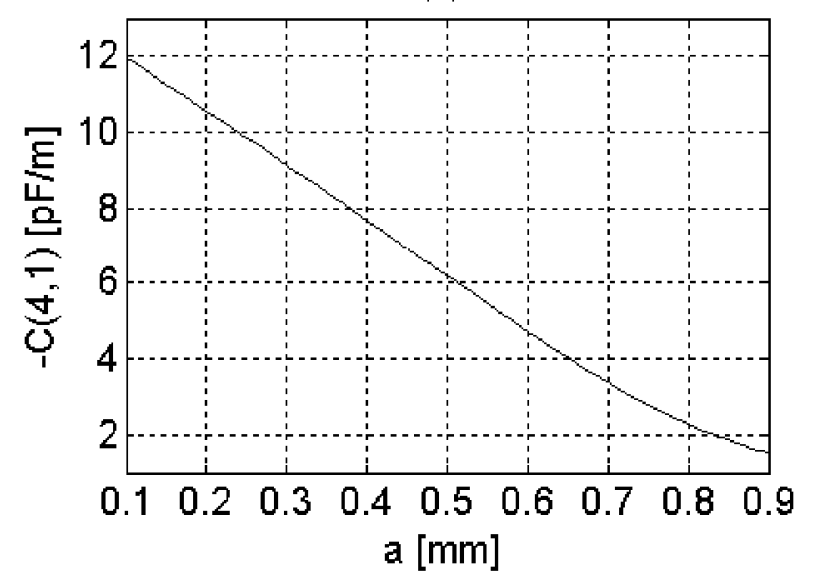

(b)

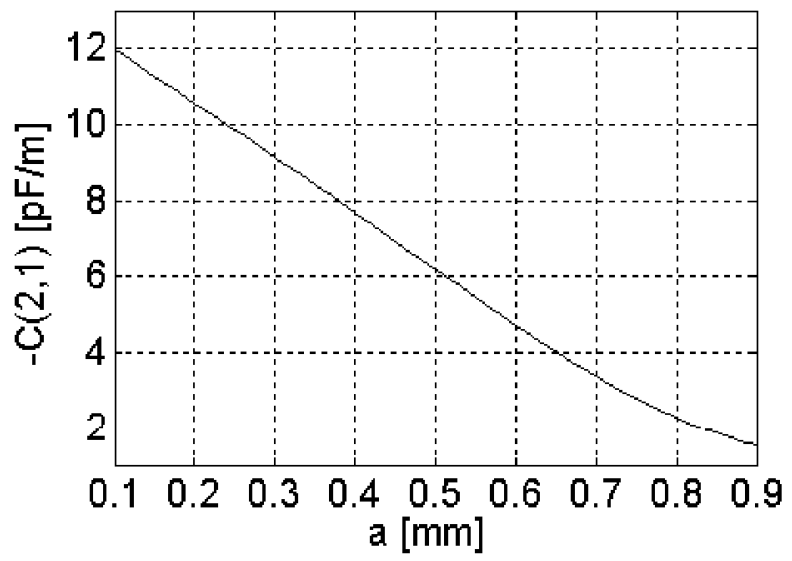

(d)

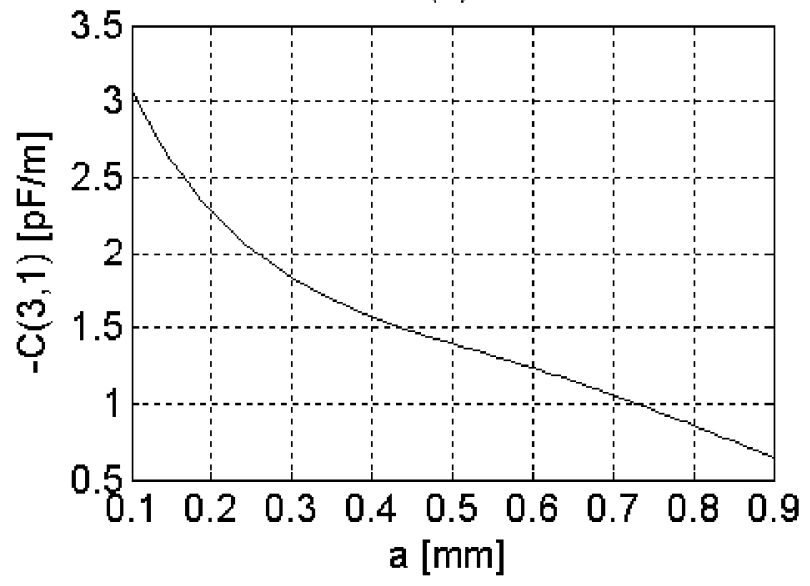

Figure (5): The capacitance coefficients versus a for $b=1.0 \mathrm{~mm}$ and $r h=0.5$ 


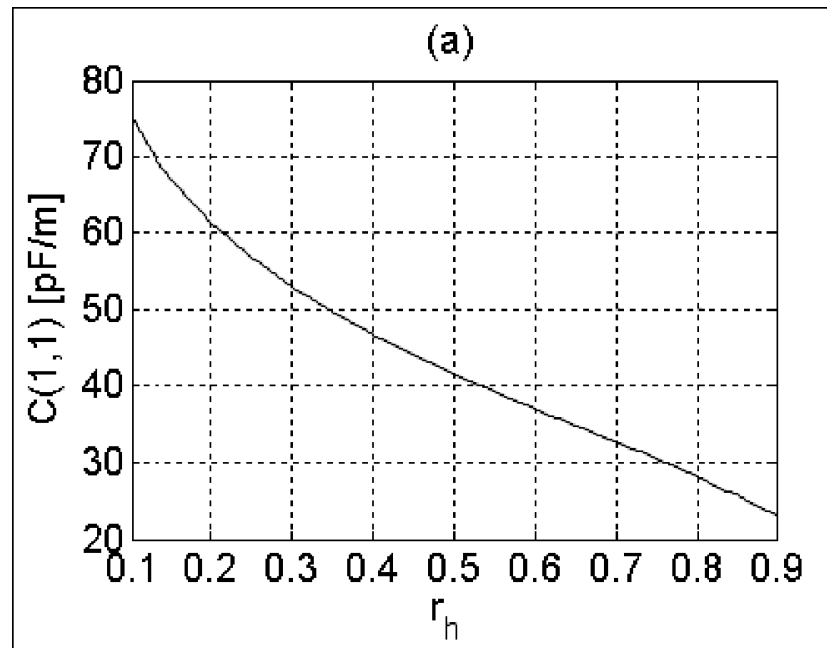

(c)

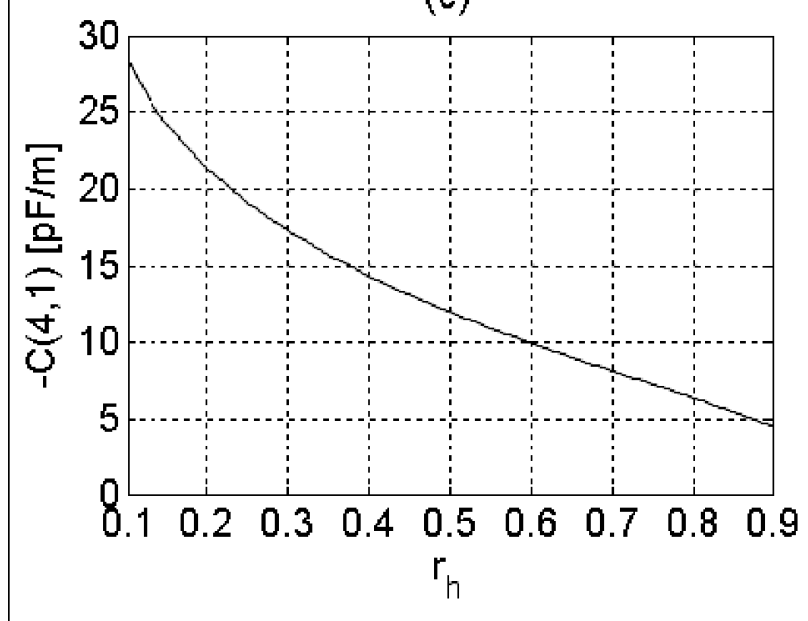

(b)

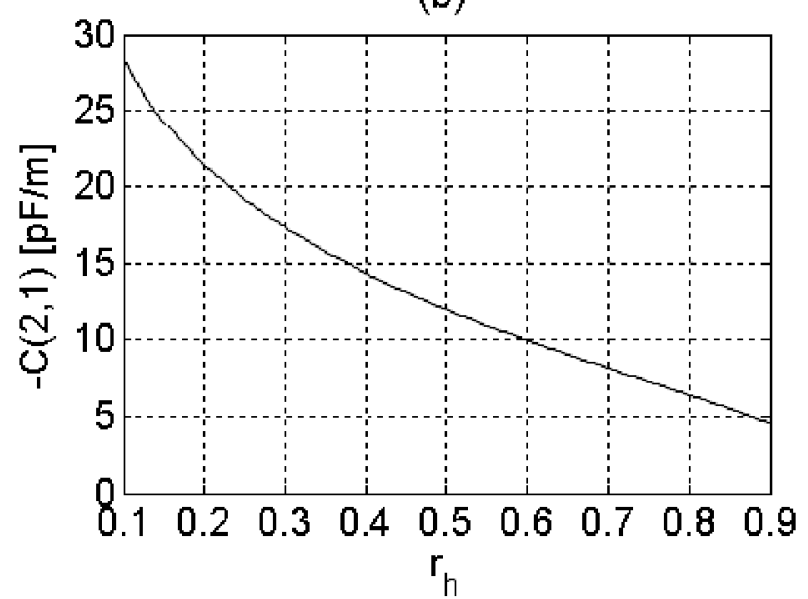

(d)

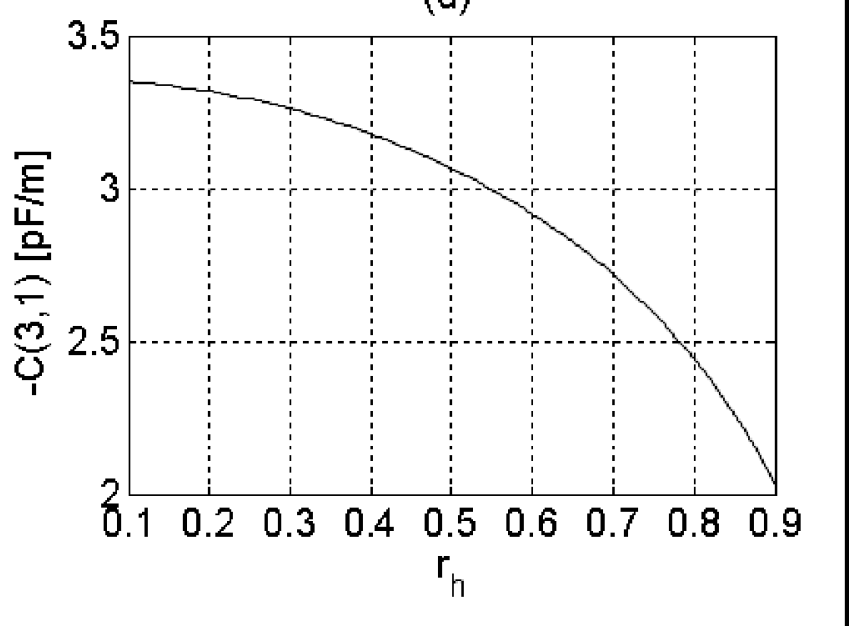

Figure (6): The capacitance coefficients versus $r$ for $a=0.1 \mathrm{~mm}$ and $b=1.0 \mathrm{~mm}$

\section{References:}

[1] C. R. Paul, "Analysis of multiconductor transmission lines”, John Wiley and Sons Inc., 1994.

[2] H. A. Wheeler, "Transmission line properties of parallel wide strips by a conformal mapping approximation”, IEEE Trans. Microwave Theory Tech., pp. 280-289, May 1964.

[3] C. Wan, "Analitically and accurately determined quasi-static parameters of coupled microstrip lines", IEEE Trans. Microwave Theory Tech., pp. 75-79, Jan. 1996.

[4] E. Yamashita and R. Mittra, "Variational method for the analysis of microstrip lines”, IEEE Trans. Microwave Theory Tech., pp. 251-256, Apr. 1968. 
[5] J. W. Duncan, "Characteristic impedances of multiconductor strip transmission lines”, IEEE Trans. Microwave Theory Tech., pp. 107-118,Jan. 1965.

[6] T. Itoh and R. Mittra, "A technique for computing dispersion characteristics of shielded microstrip lines", IEEE Trans. Microwave Theory Tech., pp. 896-898, Oct. 1974.

[7] A. Sawicki and K. Sachse, "Lower and upper bound calculation on the apacitance of multiconductor printed transmission lines and using the spectral-domain approach and variational method", IEEE Trans. Microwave Theory Tech., pp. 236-244, Feb. 1986.

[8] T. G. Bryant and J. A. Weiss, "Parameters of microstrip transmission lines and of coupled pairs of microstrip lines”, IEEE Trans.Microwave Theory Tech., pp. 0211027, Dec.1968.

[9] W. T. Weeks, "Calculation of coefficients of capacitance of multiconductor ransmission lines in the presence o a dielectric interface”, IEEE Trans. Microwave Theory Tech., pp. 35-43, Jan. 1970.

[10] D. Homentcovschi, and R. Oprea, "Analytically determined quasi-static parameters of shielded or open multiconductor microstrip lines", IEEE Trans. MicrowaveTheory Tech., pp. 18-24, Jan. 1998.

[11] M. Khalaj Amirhosseini, "Determination of capacitance and conductance matrices of lossy shielded coupled microstrip transmission lines", Progress in Electromagnetics Research, PIER 50, pp. 267-278, 2005. 